\title{
Equality and Identity of Fuzzy Numbers and Fuzzy Arithmetic with Equality Constraints
}

\author{
Sizong Guo ${ }^{1}$ Haitao Liu ${ }^{2}$ \\ ${ }^{1}$ College of Science, Liaoning Technical University, Fuxin 123000, P.R.China \\ ${ }^{2}$ College of Science, Liaoning Technical University, Fuxin 123000, P.R.China
}

\begin{abstract}
The difference between equality and identity of two fuzzy numbers is discussed in this paper. According to fuzzy arithmetic with requisite constraints put forward by Klir, the fuzzy arithmetic with equality constraints and its expression based on structured element are put forward, and then the irreversible problem in traditional fuzzy arithmetic is solved. From the expression based on structured element, the fuzzy arithmetic with equality constraints is turned into the operation of two monotone functions with the same monotonic form. From this transition we get the operation method of equality constraint arithmetic and the calculation method of fuzzy equation.
\end{abstract}

Keywords: Fuzzy numbers, Identity, Generalized constraint operators, Fuzzy equation

\section{The problems in fuzzy arith- metic}

The set of all bounded closed fuzzy numbers on real number field $R$ is denoted by $\tilde{N}_{C}(R)$. If $\tilde{A} \in \tilde{N}_{C}(R)$, then the membership function of $\tilde{A}$ is $\mu_{\tilde{A}}(x), x \in R$.

Let $(G, *)$ be a group. If $a, b \in G$, and $a * b^{-1}=$ $a \bar{*} b$, then $\bar{*}$ is called the inverse operator of $*$, where $b^{-1}$ is the inverse element of $b$.

As is well known, the fuzzy arithmetic is defined by extension principle[1, 2, 4]. For example, $\tilde{A}, \tilde{B}$, are the given bounded closed fuzzy numbers and $*$ is a binary operator. From extension principle, the membership function of $\tilde{A} * \tilde{B}$ is

$$
\mu_{\tilde{A} * \tilde{B}}(z)=\underset{z=x * y}{\vee}\left[\mu_{\tilde{A}}(x) \wedge \mu_{\tilde{B}}(y)\right]
$$

But if we use extension principle without analysis in fuzzy arithmetic, it often leads to dubious results. Now there exist at least three problems in fuzzy arithmetic.
(I) The problem in the arithmetic process between one fuzzy number and itself

Let $\tilde{A}, \tilde{B} \in \tilde{N}_{C}(R)$. From the definition of fuzzy arithmetic (1), if $\tilde{A}=\tilde{B} \neq$ constant, then $\tilde{A}-\tilde{B} \neq 0$, and $\tilde{A}-\tilde{A} \neq 0, \tilde{A} \div \tilde{A} \neq 1$. In the application, it is difficult to accept.

(II) The problem in multiplication and power operation of fuzzy numbers

From the extension principle the membership functions of $\tilde{A} \cdot \tilde{A}$ and $\tilde{A}^{2}$ are separately

$$
\mu_{\tilde{A} \cdot \tilde{A}}(z)=\bigvee_{z=x \cdot y}\left[\mu_{\tilde{A}}(x) \wedge \mu_{\tilde{A}}(y)\right]
$$

and

$$
\mu_{\tilde{A}^{2}}(z)=\underset{z=x^{2}}{\bigvee} \mu_{\tilde{A}}(x)
$$

So generally speaking, $\mu_{\tilde{A} \cdot \tilde{A}}(z) \neq \mu_{\tilde{A}^{2}}(z)$, and then $\tilde{A} \cdot \tilde{A} \neq \tilde{A}^{2}$.

(III) The irreversible problem of fuzzy arithmetic

Let $(R, *)$ be a group, and $\bar{*}$ is the inverse operator of $*$. For the given bounded closed fuzzy numbers $\tilde{A}, \tilde{B}, \tilde{C}$, if $\tilde{A} * \tilde{B}=\tilde{C}$, and $\tilde{A}$ is solvable, then we can not obtain $\tilde{A}=\tilde{C} \bar{*} \tilde{B}$. Conversely, if $\tilde{A}=\tilde{C} \bar{*} \tilde{B}$, we can not obtain $\tilde{A} * \tilde{B}=\tilde{C}$.

From the problems it follows that there exists essential difference between fuzzy arithmetic and ordinary real number arithmetic, and fuzzy arithmetic is more complex than ordinary real number arithmetic.

To overcome the problems in I and II in the applications, Klir [5] puts forward fuzzy arithmetic with requisite constraints. For the sake of simplicity, we call it constraint arithmetic. Let $A_{\lambda}$ be the $\lambda$-cut of fuzzy number $\tilde{A}$, i.e. $A_{\lambda}=\left\{x \mid \mu_{\tilde{A}}(x) \geq\right.$ $\lambda, x \in R\}$. For the binary operator $*$, the corresponding constraint operator $*_{R}$ is defined as

$$
\left(\tilde{A} *_{R} \tilde{A}\right)_{\lambda}=A_{\lambda} *_{R} A_{\lambda}=\left\{x * x \mid x \in A_{\lambda}\right\}
$$

If $*$ is a division operator, then $0 \notin A_{\lambda}$

$$
\tilde{A} *_{R} \tilde{A}=\bigcup_{\lambda \in[0,1]} \lambda \wedge\left(A_{\lambda} *_{R} A_{\lambda}\right)
$$


From (2) and (3) it follows that

$$
\begin{aligned}
& \tilde{A}-{ }_{R} \tilde{A}=0, \quad \tilde{A} \cdot{ }_{R} \tilde{A}=\tilde{A}^{2}, \\
& \tilde{A} \div{ }_{R} \tilde{A}=1,\left(0 \notin A_{\lambda}, \forall \lambda>0\right),
\end{aligned}
$$

But the constraint arithmetic only takes the arithmetic of identical fuzzy numbers into account. For $\tilde{A} * \tilde{B}=\tilde{C}$, we still can not get $\tilde{A}=\tilde{C} \bar{*} \tilde{B}$. The constraint arithmetic can not resolve inverse operation of fuzzy numbers, so it can not solve the equation which contains fuzzy numbers.

\section{Equality and identity of two fuzzy numbers}

In order to resolve the problem of fuzzy arithmetic which contains equal fuzzy numbers and apply the constraint arithmetic of fuzzy numbers correctly, it is necessary to put forward the definition of identity of fuzzy numbers and understand the difference between equality and identity of two fuzzy numbers. For sake of understanding, we use intervals as example. $\bar{A}, \bar{B}$, are intervals, and their operation is defined as

$$
\bar{A} * \bar{B}=\{z \mid z=x * y, \quad x \in \bar{A}, y \in \bar{B}\}
$$

If $*$ is a division operator, then $0 \notin \bar{B}$

Let $\bar{A}=[1,2], \bar{B}=[1,2]$, the subtraction and division are

$$
\begin{gathered}
\bar{A}-\bar{B}=[1,2]-[1,2]=[-1,1] \\
\bar{A} \div \bar{B}=[1,2] \div[1,2]=[1,2] \cdot[1 / 2,1]=[1 / 2,2]
\end{gathered}
$$

Although $\bar{A}$ and $\bar{B}$ have the same interval, they are not identical in essence, and $\bar{A}$ and $\bar{B}$ are called equality. If $\bar{A}$ and $\bar{B}$ are identical, then

$$
\begin{aligned}
& \tilde{A}-{ }_{R} \tilde{A}=[0,1]-{ }_{R}[0,1]=0, \\
& \tilde{A} \div_{R} \tilde{A}=[0,1] \div{ }_{R}[0,1]=1 .
\end{aligned}
$$

The two intervals in the expressions above are identical, and we can use constraint arithmetic. So the constraint arithmetic which is put forward by Klir is fit for the operations between the identical intervals, not between the equal intervals. Based on the work above, we call the constraint arithmetic which is put forward by Klir identity constraint arithmetic.

In fuzzy arithmetic the definition of identity of fuzzy numbers is very important. For the equal fuzzy numbers, we calculate them with (1); for the identical fuzzy numbers, we calculate them with (2) and (3). In fuzzy analysis the definition of identity of fuzzy numbers is also very important. For example, the fuzzy-value function are

$$
\tilde{f}(x)=\tilde{\lambda} e^{-\tilde{\beta} x}, \tilde{g}(x)=\tilde{\lambda} e^{-\tilde{\lambda} x}
$$

where $\tilde{\lambda}$ and $\tilde{\beta}$ are fuzzy numbers. In the fuzzyvalue function $\tilde{f}(x)$, although fuzzy parameters $\tilde{\lambda}$ and $\tilde{\beta}$ may have the equal fuzzy numbers, they are different in essence and independent from each other. However, in the fuzzy-value function $\tilde{g}(x)$, the two fuzzy parameters are identical and correlative. So the two fuzzy-value functions $\tilde{f}(x)$ and $\tilde{g}(x)$ are different in essence, and we should make use of identity constraint arithmetic to solve the membership function of $\tilde{g}(x)$.

\section{The structured element ex- pression of identity con- straint arithmetic}

The expression of structured element of fuzzy number is put forward in [6]. According to the homeomorphic property $[7,8]$ between the monotone function family with the same monotonic form on $[-1$, $1]$ and the bounded fuzzy number, we can easily get the equivalent representation of identity constraint arithmetic on the monotone function family with same monotonic form. On the work above we extend the identity constraint arithmetic to the equality constraint arithmetic in which the fuzzy numbers is equal, and then resolve the inverse operation of fuzzy numbers.

Let's review some definitions and theorems about structured element[6].

Let $E$ be a fuzzy set on the real number field $R$ and $E(x)$ is the membership function of $E$. We call that $E$ is a fuzzy structured element on $R$, if it satisfies the following properties

1) $E(0)=1 ; 2) E(x)$ is the monotone increasing and right continuous function on $[-1,0]$, the monotonic decreasing and left continuous on $(0,1]$; 3) $E(x)=0(-\infty<x<-1$ and $1<x<+\infty)$.

Let $E$ be a fuzzy structured element, and it satisfies the following properties: (i) $\forall x \in(-1,1)$, $E(x)>0$; (ii) $E(x)$ is continuous and strictly monotone increasing on $[-1,0]$ and continuous and strictly monotone decreasing on $(0,1]$, then $E$ is said to be a regular fuzzy structured element. If $E(-x)=E(x)$, then $E$ is said to be a symmetrical fuzzy structured element.

Theorem 3.1 [6] Let $E$ be an arbitrary fuzzy structured element, $E(x)$ be its membership function, $f(x)$ be a monotone function on $[-1,1]$, and 
$\hat{f}(x)$ be the set-valued extension function of $f(x)$, then $\hat{f}(E)$ is a bounded closed fuzzy number, and the membership function of $\hat{f}(E)$ is $E\left(f^{-1}(x)\right)$, where $\hat{f}^{-1}(x)$ is the rotation symmetric function of $f(x)$ about variables $x$ and $y$ (If $f^{-1}(x)$ is continuous and strictly monotone, then $f^{-1}(x)$ is the inverse function of $f(x))$.

The definition of set-valued extension function is put forward in [6]. In the following, we denote $\hat{f}(x)$ as $f(x)$.

Let $\lambda$-cuts of fuzzy structured element $E$ be $E_{\lambda}=\{x \mid E(x) \geq \lambda\}=\left[e_{\lambda}^{-}, e_{\lambda}^{+}\right]$for all $\lambda \in(0,1]$. From the definition of fuzzy structured element it follows that $e_{\lambda}^{-} \in[-1,0], e_{\lambda}^{+} \in[0,1]$.

Theorem 3.2 [6] If $E$ is a given regular fuzzy structured element and $\tilde{A}$ is an arbitrary bounded closed fuzzy number, then there must exist a monotone function $f(x)$ in $[-1,1]$, such that $\tilde{A}=$ $f(E)$. If $f(x)$ is monotone increasing on $[-1,1]$, then the $\lambda$-cuts of $\tilde{A}$ is

$$
A_{\lambda}=f\left(E_{\lambda}\right)=\left[f\left(e_{\lambda}^{-}\right), f\left(e_{\lambda}^{+}\right)\right]
$$

for all $\lambda \in(0,1]$; if $f(x)$ is monotone decreasing on $[-1,1]$, then the $\lambda$-cuts of $\tilde{A}$ is

$$
A_{\lambda}=\left[f\left(e_{\lambda}^{+}\right), f\left(e_{\lambda}^{-}\right)\right] .
$$

The proofs of theorem 3.1 and 3.2 are put forward in [6].

Theorem 3.3 Let $E$ be an arbitrary fuzzy structured element, $f(x)$ be a monotone function on [-1, 1]. If $\tilde{A}=f(E)$, then the identity constraint arithmetic operator $*_{R}$ of binary operator $*$ is equivalent to

$$
\tilde{A} *_{R} \tilde{A}=[f(x) * f(x)]_{x=E}=[f * f](E),
$$

and the membership function of $\tilde{A} *_{R} \tilde{A}$ is

$$
\mu_{\tilde{A} *_{R} \tilde{A}}(x)=E\left[(f * f)^{-1}(x)\right]
$$

If $*$ is a division operator, then $f(x) \neq 0, x \in$ $[-1,1]$.

Proof Let $\tilde{A}=f(E)$. By means of (5) we have

$$
\left(\tilde{A} *_{R} \tilde{A}\right)_{\lambda}=[(f * f)(E)]_{\lambda}=(f * f)\left(E_{\lambda}\right)
$$

Let $F(x)=f(x) * f(x)$, then $\left(\tilde{A} *_{R} \tilde{A}\right)_{\lambda}=F\left(E_{\lambda}\right)$

From (6) it follows that for all $y \in F\left(E_{\lambda}\right)$ there exists $x \in E_{\lambda}$, such that $y=f(x) * f(x)$ and $f(x) \in$ $f\left(E_{\lambda}\right)=A_{\lambda}$. Let $z=f(x)$, then

$\left(\tilde{A} *_{R} \tilde{A}\right)_{\lambda}=F\left(E_{\lambda}\right)=\left\{z * z \mid z=f(x) \in f\left(E_{\lambda}\right)=A_{\lambda}\right\}$ which proves theorem 3.3.

From the theorem 3.3 it follows that the equivalent expression of fuzzy structured element of identity constraint arithmetic $*_{R}$ is the operation of monotone functions, there no needs any transitions of monotone functions[7]. In identity constraint arithmetic, if the two fuzzy numbers are identical, then the monotone functions corresponding are identical. In the following, we extend (5) to the situation that the two monotone functions are not identical.

Definition 3.1 Let $E$ be an arbitrary fuzzy structured element, $f(x)$ and $g(x)$ be monotone functions with the same monotonic form on $[-1,1]$. If $\tilde{A}=f(E), \tilde{B}=g(E), *$ is a binary operator on $R$, and $f(x) * g(x)$ has the same monotonic form with $f$ and $g$ on $[-1,1]$, then $\bar{*}_{R}$ is the generalized constraint operator of $*$. If

$$
\tilde{A} *_{R} \tilde{B}=[f(x) * g(x)]_{x=E}=[f * g](E)
$$

then the membership function of $\tilde{A} *_{R} \tilde{B}$ is

$$
\mu_{\tilde{A} *_{R} \tilde{B}}(x)=E\left[(f * g)^{-1}(x)\right] .
$$

The set of all monotone functions with the same monotonic form on $[-1,1]$ is denoted by $\mathrm{D}[-$ 1,1]. In [7], the definition of monotone transition with the same monotonic form is

$$
\begin{aligned}
& \tau_{i}: D[-1,1] \rightarrow D[-1,1], \\
& i=0,1,2,3, \text { for all } f \in D[-1,1],
\end{aligned}
$$

Let

$$
\begin{aligned}
& \tau_{0}(f)=f ; \tau_{1}(f)=f^{\tau_{1}} ; \\
& \tau_{2}(f)=f^{\tau_{2}} ; \tau_{3}(f)=f^{\tau_{3}} .
\end{aligned}
$$

Where $f^{\tau_{1}}(x)=-f(-x) ; f^{\tau_{2}}(x)=\frac{1}{f(-x)}$, $(f(-x) \neq 0) ; f^{\tau_{3}}(x)=-\frac{1}{f(x)},(f(x) \neq 0)$, for all $\mathrm{x} \in[-1,1]$.

Let $T=\left\{\tau_{0}, \tau_{1}, \tau_{2}, \tau_{3}\right\}$, the multiplication on $T$ is $\tau_{i} \tau_{j}(f)=f^{\tau_{i} \tau_{j}}=\left(f^{\tau_{j}}\right)^{\tau_{i}}$. It is easy to prove that $T$ is a commutative group about the definition above, and we call it transformation group with the same monotonic form of monotone functions.

Lemma 3.1 Let $E$ be a fuzzy structured element, if $f$ and $g$ are monotone functions with the same monotonic form on $[-1,1]$, and $\tilde{A}=f(E), \tilde{B}=$ $g(E)$, then

(a) If $\tilde{A}$ and $\tilde{B}$ are arbitrary bounded fuzzy numbers, then $\tilde{A}+\tilde{B}=(f+g)(E)$, and its membership function is

$$
\mu_{\tilde{A}+\tilde{B}}(x)=E\left((f+g)^{-1}(x)\right) ;
$$


(b) If $\tilde{A}$ and $\tilde{B}$ are both positive fuzzy numbers, then $\tilde{A} \cdot \tilde{B}=f(E) \cdot g(E)=[f \cdot g](E)$, and its membership function is

$$
\mu_{\tilde{A} \cdot \tilde{B}}(x)=E\left[(f \cdot g)^{-1}(x)\right] .
$$

Lemma 3.2 Let $E$ be a symmetrical fuzzy structured element, and $f$ is a monotone bounded function on $[-1,1]$. If $\tilde{A}=f(E)$, then

$$
\begin{aligned}
& -\tilde{A}=f^{\tau_{1}}(E) ; \quad \frac{1}{\tilde{A}}=f^{\tau_{2}}(E) ; \\
& -\frac{1}{\tilde{A}}=f^{\tau_{1} \tau_{2}}(E) \stackrel{=}{=} f^{\tau_{3}}(E) .
\end{aligned}
$$

The proof is put forward in [7].

From the definition of generalized constraint operator $*_{R}$ it follows that:

Proposition 3.1 $\tilde{A}+{ }_{R} \tilde{B}=\tilde{A}+\tilde{B}$

Proposition 3.2 If $\tilde{A}$ and $\tilde{B}$ are positive fuzzy numbers, then $\tilde{A} \cdot_{R} \tilde{B}=\tilde{A} \cdot \tilde{B}$

From lemma 3.1 and definition 3.1 it is easy to prove the proposition 3.1 and 3.2 .

Proposition 3.3 $\tilde{A}, \tilde{B}, \tilde{C} \in \tilde{N}_{C}(R)$, $\tilde{B}$;

(a) If $\tilde{A}+\tilde{B}=\tilde{C}$, then $\tilde{C}-{ }_{R} \tilde{B}=\tilde{A}, \tilde{C}-{ }_{R} \tilde{A}=$

(b) If $\tilde{A} \cdot \tilde{B}=\tilde{C}$, and for all $\lambda>0,0 \notin B_{\lambda}$, then $\tilde{A}=\tilde{C} \div{ }_{R} \tilde{B}$;

(c) $\tilde{A}^{2}=\tilde{A} \cdot R \tilde{A}$

\section{Equality constraint arith- metic of fuzzy numbers and its structured element ex- pression}

In the following, we will discuss the inverse operation of fuzzy numbers. For sake of understanding, we also use the intervals as examples.

Example 1 Let $\bar{A}=[0,1], \bar{B}=[2,3]$. From the definition of operation of intervals it follows that $\bar{C}=\bar{A}+\bar{B}=[2,4]$, and $\bar{C}-\bar{B}=[2,4]-[2,3]$ $=[2-3,4-2]=[-1,2] \neq \bar{A}$, i.e. $\bar{A} \neq \bar{C}-\bar{B}$.

Example 2 Let $\bar{C}=[2,4], \bar{B}=[2,3]$. If $\bar{C}=\bar{A}+\bar{B}$, then $\bar{A}=$ ?

From the definition of addition of intervals it follows that $\bar{A}=[0,1]$, but $\bar{A} \neq \bar{C}-\bar{B}$, and $(\bar{C}-$ $\bar{B})+\bar{B} \neq \bar{C}$. It comes into existence for the fuzzy numbers.

The meaning which is denoted by interval $\bar{A}$ in example 1 is different from that in example 2. In example 2 , it is stressed that $\bar{A}$ is decided by $\bar{C}=\bar{A}+\bar{B}$, i.e. $\bar{A}$ is constrained by $\bar{C}=\bar{A}+\bar{B}$; in example 1, they use $\bar{C}-\bar{B}$ to solve $\bar{A}$ and it is not stressed that $\bar{A}$ is constrained by $\bar{C}=\bar{A}+\bar{B}$. It is necessary to define a more generalized constraint arithmetic so that $\bar{C}-{ }_{e} \bar{B}=\bar{A}$. What is different from the identity constraint arithmetic (1) is that the two intervals or fuzzy numbers in this operation is not identical.

Definition 4.1 Let $(R, *)$ be a group, and $₹$ is the inverse operator of $*$. For $\tilde{A}, \tilde{B}, \tilde{C} \in \tilde{N}_{C}(R)$, if $\tilde{A} * \tilde{B}=\tilde{C}$ is solvable about $\tilde{A}$, and $\tilde{A}=\tilde{C} \bar{*}_{e} \tilde{B}$, then $\bar{*}_{e}$ is called equality constraint operator of $\tilde{A} * \tilde{B}=$ $\tilde{C}$.

As is well known, the definition of Hukuhara difference[9] of fuzzy numbers is: Let $\tilde{A}, \tilde{B} \in$ $\tilde{N}_{C}(R)$, if there exists $\tilde{C} \in \tilde{N}_{C}(R)$, such that $\tilde{A}=\tilde{B}+\tilde{C}$, then $\tilde{C}$ is called Hukuhara difference of $\tilde{A}$ and $\tilde{B}$, short for $H$ difference, denoted by $\tilde{C}=\tilde{A}-{ }_{H} \tilde{B}$. Obviously, $H$ difference is the equality constraint arithmetic of addition of fuzzy numbers or the equality constraint subtraction of fuzzy numbers.

If fuzzy equation $\tilde{A} \cdot \tilde{X}=\tilde{B}$ has only one solution, and it is denoted by $\tilde{X}=\tilde{B} \div{ }_{e} \tilde{A}$, then $\div e$ is the equality constrained division of equation $\tilde{A} \cdot \tilde{X}=\tilde{B}$.

It is necessary to give an illustration: if $\tilde{D}=$ $-\tilde{B}, \tilde{D}^{\prime}=\frac{1}{\tilde{B}}$, then $\tilde{A}-\tilde{B}=\tilde{C}$ can be seen as $\tilde{A}+\tilde{D}=\tilde{C}$, and $\tilde{A} \div \tilde{B}=\tilde{C}$ can be seen as $\tilde{A}$. $\tilde{D}^{\prime}=\tilde{C}$. By means of lemma 3.2 it is easy to get the structured element expressions of $\tilde{D}$ and $\tilde{D}^{\prime}$. So we only discuss the equations $\tilde{A}+\tilde{X}=\tilde{C}$ and $\tilde{A} \cdot \tilde{X}=\tilde{B}$.

The following theorems imply that the equality constraint arithmetic and generalized constraint arithmetic of equations $\tilde{A}+\tilde{X}=\tilde{C}$ and $\tilde{A} \cdot \tilde{X}=\tilde{B}$ are equivalent.

Theorem 4.1 Let $E$ be an arbitrary fuzzy structured element, $f$ and $g$ be the monotone functions with the same monotonic form on $[-1,1] . \tilde{A}=$ $f(E), \tilde{B}=g(E)$. If $\tilde{A}+\tilde{X}=\tilde{B}$ is solvable about $\tilde{X}$, then $\tilde{X}=\tilde{B}-{ }_{R} \tilde{A}$, and $-_{R}$ is the generalized constraint operator of subtraction operator -, i.e.

$$
\tilde{X}=h(E), \text { where } h(x)=g(x)-f(x),
$$

for all $x \in[-1,1]$. and the membership function of $\tilde{X}$ is

$$
\mu_{\tilde{X}}(x)=E\left[(g-f)^{-1}(x)\right]
$$

Proof Let $\tilde{X}^{\prime}=h(E)$, and $h(x)=g(x)-f(x)$. We need to prove that $\tilde{A}+\tilde{X}^{\prime}=\tilde{B}$. By means of the 
structured element expression of fuzzy arithmetic we have

$$
\tilde{A}+\tilde{X}^{\prime}=f(E)+h(E)
$$

From lemma 4.1(a) it follows that

$$
\begin{aligned}
\mu_{\tilde{A}+\tilde{X}^{\prime}}(x) & =E\left((f+h)^{-1}(x)\right) \\
& =E\left((f+g-f)^{-1}(x)\right) \\
& =E\left(g^{-1}(x)\right)=\mu_{\tilde{B}}(x)
\end{aligned}
$$

which proves that $\tilde{A}+\tilde{X}^{\prime}=\tilde{B}$.

Theorem 4.2 Let $E$ be an arbitrary fuzzy structured element. $\tilde{A}=f(E), \tilde{B}=g(E)$, where $f$ and $g$ are the monotone functions with the same monotonic form on $[-1,1]$, and for all $x \in[-1,1]$, $f(x)>0, g(x)>0$. If $\tilde{A}+\tilde{X}=\tilde{B}$ is solvable about $\tilde{X}$, then $\tilde{X}=\tilde{B} \div_{R} \tilde{A}$, and $\div_{R}$ is the generalized constraint operator of real number division $\div$, i.e.

$$
\tilde{X}=h(E), \text { where } h(x)=g(x) \div f(x),
$$

for all $x \in[-1,1]$, and the membership function of $\tilde{X}$ is

$$
\mu_{\tilde{X}}(x)=E\left[(g \div f)^{-1}(x)\right] .
$$

Making use of the similar proof of theorem 4.1 and lemma 4.1 it is easy to prove theorem 4.2.

Theorem 4.3 The necessary and sufficient condition that $\tilde{A}+\tilde{X}=\tilde{B}$ is solvable is

$$
a_{2}(\lambda)-b_{2}(\lambda) \leq a_{1}(\lambda)-b_{1}(\lambda), \text { for all } \lambda \in(0,1],
$$

where $A_{\lambda}=\left[a_{1}(\lambda), a_{2}(\lambda)\right], B_{\lambda}=\left[b_{1}(\lambda), b_{2}(\lambda)\right]$ are the $\lambda$-cuts of $\tilde{A}$ and $\tilde{B}$.

Proof Let $X_{\lambda}=\left[x_{1}(\lambda), x_{2}(\lambda)\right]$. If $\tilde{A}+\tilde{X}=\tilde{B}$ is solvable, then for all $\lambda \in(0,1]$

$$
\left[a_{1}(\lambda), a_{2}(\lambda)\right]+\left[x_{1}(\lambda), x_{2}(\lambda)\right]=\left[b_{1}(\lambda), b_{2}(\lambda)\right],
$$

and

$$
b_{1}(\lambda)-a_{1}(\lambda)=x_{1}(\lambda) \leq x_{2}(\lambda)=b_{2}(\lambda)-a_{2}(\lambda) .
$$

So

$$
a_{2}(\lambda)-b_{2}(\lambda) \leq a_{1}(\lambda)-b_{1}(\lambda) .
$$

Theorem 4.4 Let $E$ be a symmetrical fuzzy structured element. $\tilde{A}=f(E), \tilde{B}=g(E)$, and $f$ and $g$ are the monotone functions with the same monotonic form on $[-1,1]$. The necessary and sufficient condition that $\tilde{A}+\tilde{X}=\tilde{B}$ is solvable is

$$
f(x)-g(x) \leq f(-x)-g(-x), \text { for all } x \in[0,1]
$$

Proof Suppose that $f$ and $g$ are monotone increasing functions on $[-1,1]$, then for all $\lambda \in(0,1]$,

$$
\begin{aligned}
& A_{\lambda}=f\left(E_{\lambda}\right)=\left[f\left(e_{\lambda}^{-}\right), f\left(e_{\lambda}^{+}\right)\right], \\
& B_{\lambda}=g\left(E_{\lambda}\right)=\left[g\left(e_{\lambda}^{-}\right), g\left(e_{\lambda}^{-}\right)\right],
\end{aligned}
$$

and from theorem 4.3 the necessary and sufficient condition that $\tilde{A}+\tilde{X}=\tilde{B}$ is solvable is

$$
f\left(e_{\lambda}^{+}\right)-g\left(e_{\lambda}^{+}\right) \leq f\left(e_{\lambda}^{-}\right)-g\left(e_{\lambda}^{-}\right),
$$

for all $\lambda \in(0,1]$. $E$ is a symmetrical fuzzy structured element, then for all $\lambda \in(0,1]$ we have $e_{\lambda}^{+} \in[0,1], e_{\lambda}^{-} \in[-1,0]$, and $\left|e_{\lambda}^{-}\right|=e_{\lambda}^{+}$. Let $e_{\lambda}^{+}=x, e_{\lambda}^{-}=-x$, and from (8) it follows that $f(x)-g(x) \leq f(-x)-g(-x)$ which proves theorem 4.4 .

Theorem 4.5 The necessary and sufficient condition that $\tilde{A}+\tilde{X}=\tilde{B}$ is solvable is

$$
\frac{b_{1}(\lambda)}{a_{1}(\lambda)} \leq \frac{b_{2}(\lambda)}{a_{2}(\lambda)}, \text { for all } \lambda \in(0,1] .
$$

If $E$ is a symmetrical fuzzy structured element, $\tilde{A}=$ $f(E), \tilde{B}=g(E)$, and $f$ and $g$ are the monotone functions with the same monotonic form on [-1, 1], then the necessary and sufficient condition that $\tilde{A}+\tilde{X}=\tilde{B}$ is solvable is

$$
\frac{g(-x)}{f(-x)} \leq \frac{g(x)}{f(x)}, \text { for all } x \in[0,1] .
$$

The proof is similar with the proof of theorem 4.4 .

\section{Conclusions}

The equality constraint arithmetic is put forward based on the identity constraint arithmetic of Klir, and the difference between these two constraint arithmetic is that in the equality constraint arithmetic fuzzy numbers are not identical. According to the structured element expression of fuzzy numbers, the equality constraint arithmetic is turned into the operation of two monotone functions with the same monotonic form. From this transition we can get operation method of equality constraint arithmetic, and reasonable explanation and calculation method of solution of fuzzy equation.

\section{References}

[1] L.A. Zadeh, Fuzzy Sets. Information and Control, 8:338-353, 1965. 
[2] S.S. Chang and L.A. Zadeh, On fuzzy mapping and control. IEEE Trans. Syst., Man Cybern, 2:30-34, 1972.

[3] D. Dubois and H. Prade, Comment on "Tolerance analysis using fuzzy sets" and "A procedure for multiple aspect decision making". Int. J. Syst. Sci, 9(3):357-360, 1978.

[4] C.Z. Luo, Theory of fuzzy sets, Beijing Normal University Press, Beijing, 1994.

[5] G.J. Klir, Fuzzy arithmetic with requisite constraints. Fuzzy Sets and Systems, 91:165-175, 1997.

[6] S.Z. Guo, Z.X. Su and L. Wang, Method of Structured Element in Fuzzy Analysis and Calculation. Fuzzy System and Mathematics, 18(3):68-75, 2004.

[7] S.Z. Guo, Transformation group of monotone functions with same monotonic form on $[-1,1]$ and operations of fuzzy numbers. Fuzzy System and Mathematics, 19(3):105-110, 2005.

[8] S.Z. Guo, Homeomorphic property between fuzzy number space and family of standard bounded monotone function. Progress in Natural Science, 14(11):1318-1321, 2004.

[9] C.X. Wu and M. Ma, Basic on fuzzy analysis, National Defence industry Press, Beijing, 1991. 